\title{
Homeopathy of Mucormycosis
}

\author{
Dr. Mandeep Singh \\ PG Scholar, Department of Homoeopathic Materia Medica \\ U/G/O Dr. Sahil Mittu MD (Hom) \\ Sri Guru Nanak Dev Homoeopathic Medical College and Hospital, Ludhiana, Punjab, India
}

\begin{abstract}
The article "Mucormycosis and Homeopathic Approach" will help in understanding the disease condition and its treatment using homoeopathic remedies. Clinical features of the disease are explained on the basis of their site of infection that helps in early diagnosis and prevention of further complications.
\end{abstract}

Keywords: Mucormycosis and its homoeopathic therapeutics

\section{Introduction ${ }^{1}$}

Mucormycosis is uncommon invasive fungal infection. It is one of the more serious, aggressive and lethal invasive mycoses. The patients which are suffering from diabetes mellitus or immunocompromise diseases having more chance to infection with the Mucorales. Timely diagnosis is critical to survival and minimization of morbidity.

\section{Pathogenesis $^{1}$}

Mucorales are found commonly in the environment and spores of these usually nonpathogenic fungi are likely to be inhaled daily. In normal human lungs, spores are inhibited from germinating into hyphae by alveolar macrophages. However in diabetic patients, especially those with elevated blood sugar levels and acidemia, the spores germinate, hyphae develop and the fungi begin an in exorable march throughout the lung tissue, invading blood vessels and surrounding tissue. As the blood vessels involved, thrombosis occurs, tissue necrosis results, and the fungi continue to grow in this devitalized tissue. The use of deferoxamine to treat iron overload is a risk factor for mucormycosis; the siderophore supplies the fungi with iron that enhances their growth.

\section{Clinical features ${ }^{2}$}

The clinical manifestation depends upon the site of infection.

- Rhinocerebral mucormycosis: It starts as sinusitis with intense headache, bloody nasal discharge, changes in mental status, black eschar of the palate (hallmark of rhinocerebral mucormycosis), which indicates angioinvasion and tissue necrosis. Involvement of orbit leading to proptosis, ptosis, invasion of retinal artery causing blindness, progression of infection into the brain, and cavernous sinus thrombosis (ominous sign). CT/ MRI reveal sinus opacification and destruction of contiguous bone with brain involvement.

- Pulmonary mucormycosis: Sever progressive tissue destructive pneumonia with high fever is the common sign. Cavitations of involved lung and haematogenous spread to other organs including brain.

- Gastrointestinal mucormycosis: It is more common in patients with protein - calorie malnutrition and usually present as perforated viscus.
- Cutaneous mucormycosis: Infection follows traumatic injuries. Area of tissue necrosis enlarges rapidly involving all layers of skin and underlying structures.

Investigations $^{2}$

- There are non pathogonomic hematologic changes. The main abnormality is the underlying predisposing disorder that is diabetic ketoacidosis.

- There are elevated white blood cell counts and acute phase reactant levels. Blood culture is usually negative.

- Biopsy with histological examination is required for diagnosis.

\section{Homoeopathic management for mucormycosis}

There are some characteristic medicines which would be used on the basis of symptoms similarities to treat the mucormycosis.

Arsenicum Album ${ }^{3}$

- Burning pain, the parts burn like fire.

- Gangrenous aphthae, which burns like fire.

- All the symptoms worse at night, particularly after midnight.

- Great anguish, extreme restlessness, and fear of death.

- Great thirst for cold water, drinks very often, but takes but little at a time.

Aurum Metallicum ${ }^{3}$

- Through the organic nervous system it especially acts upon the palatine bones; osseous system, more especially the nasal and palatine bones.

- Caries of the nasal, palatine, mastoid, and ossicular bones. - RAUE.

- Swelling of the skull bones.

- Caries of bones, paining worse at night.

- Aggravation in the morning; on getting cold; while reposing.

- Relieved by moving; while walking, and on getting warm.

Condurango 5

- PAINFUL CRACKS IN CORNER OF MOUTH is a guiding symptom of this drug. Chronic gastric catarrh, syphilis, and cancer. Tumors; stricture of oesophagus. 
- Fissures form about the muco - cutaneous outlets. Epithelioma of lips or anus. Ulcerative stage of carcinoma cutis when fissures form.

Acidum Fluoricum ${ }^{3}$

- This acid is the most caustic and highly corrosive substance known.

- Caries in consequence of syphilis, or abuse of Mercury, especially of the temporal bone. - RAUE.

- Digestive Organs. - Rapid caries of the teeth.

- Dental and lachrymal fistulae.

- Syphilitic erosions, mucous tubercles, exostoses, and nightly bone - pains.

- Varicose veins; typhus with decubitus.

Sulphur Iodatum ${ }^{4}$

- Obstinate skin affections; painless enlargement of glands, and infiltration of tissues with thickening and induration after inflammation are marked features of Sul - io.

- Weakness; as from influenza. Prostration and sensitive to everything. Faint and sick. Raw burning heat internally with external coldness. Acrid discharges. Single parts, fingers etc. turn white and insensible. Suppuration.

- Breathing asthmatic, irregular, rattling and suffocative. Cough worse a. m.; spasmodic. Expectoration greenish, purulent, copious, viscid and yellow. Chest constricted, dropsy of pleura, and eruptions on chest.

- Itching. Ulcers. Moist eczema. Acne, suppurating. Barbers' itch.

- Aching soreness below knees. Soles of feet ache, burn and sore, when standing.

\section{Acidum Benzoicum ${ }^{3}$}

- Through the organic nervous system, it acts on the urinary organs, joints, fibrous tissue, and skin.

- Especially adapted to rheumatic or gouty subjects, where the urine is excessively fetid.

- The urine is high colored, with an exceedingly strong smell; dark - colored and much heavier than normal.

- Concretions in the joints, with rheumatism, or gout, with strong - smelling urine.

- Articular rheumatism, with fetid urine.

Kali Bichromicum ${ }^{3}$

- Acts through the ganglionic system upon the mucous membranes; the glandular system; fibrous tissue and skin.

- The mucous membranes chiefly affected are the mouth, throat, cardiac portion of the stomach, duodenum, jejunum, and rectum;

- The whole respiratory membrane, including the conjunctiva and the uterus.

- Upon the skin it causes papules, pustules, and ulcers.

- Upon the fibrous tissue, about the joints and the periosteum, it has a marked and powerful influence. It also affects the cartilages, especially that of the nose, which it has entirely destroyed.

Respiratory Organs:

- Cough, with expectoration of tough, stringy mucus, which sticks to the throat, mouth and lips; the cough in choking and croupy; worse in the morning.
- Discharges from the nose, mouth, throat, stomach, vagina, or any of the mucous membranes, of a tough, stringy mucus, which sticks to the parts, and can be drawn out into strings three feet long.

Mercurius Solubilis Hahnemanni ${ }^{6}$

- Complaints of Syphilitic origin.

- < heat as well as cold < night < warmth of bed.

- Nostrils are raw ulcerated. Pain and swelling of the nasal bones and decay with greenish fetid ulceration.

- Glandular enlargement with easy suppuration.

- Offensive discharges which are yellow green.

- Ulcerations spreading superficially with lardaceous base.

- Great thirst with moist mouth.

Secal Cornutum ${ }^{3}$

- Strong tendency to putrescence, discharge of black blood; a kind of sanies, with tingling in the limbs and great debility.

- Passive haemorrhages; everything seems open and loose; no action, in thin, scrawny, cachectic women.

- Dry gangrene of the extremities; the parts are dry, cold, hard, and insensible, of a uniform black color, and free from fetor. - - HEMPEL.

- The ulcer feels as though it had been burnt; discharges a putrid, bloody fluid, and is sometimes decidedly gangrenous and painless; in thin, scrawny cachectic people.

\section{References}

[1] Bennett John E, Harrison's Principles of internal medicine, Mucormycosis, McGraw Hill $2008-17^{\text {th }}$ edition - ch. 198 .

[2] Alagappan R, Manual of Practical Medicine, Clinical features mucormycosis -Jaypee BrothersMedical Publishers Pvt. Limited 2014 - $5^{\text {th }}$ edition-p.193, 194.

[3] Burt William H. Characteristic Materia Medica, University of Michigan Library - 2006.

[4] Phatak S. R. - Concise Materia Medica Of Hom. Remedies, B Jain Publishers Pvt Ltd; Enlarged Edition 2003.

[5] Boericke William - Homoeopathic Materia Medica \& Repertory, B Jain Publishers Pvt Ltd.2003.

[6] Murphy N. D. Robin - Lotus Materia Medica - B Jain Publishers Pvt Ltd.2004 - $2^{\text {nd }}$ Edition. 